\title{
Sistema de Informação em Saúde do Trabalhador: desafios e perspectivas para o SUS
}

\author{
Occupational Health Information System: challenges and \\ perspectives in the Brazilian Unified Health Sys tem (SUS)
}

Luiz Aug u s to Facchini 1

Letícia Coelho da Costa Nobre 2

Nei ce Muller Xavier Faria 1

Anaclaudia Gastal Fassa 1

Elaine Thumé 1

Elaine Tomasi 3

Vilma Santana 4

1 Departamento de Medicina Social, Faculd ade de Medicina, Universid ade Federal de Pelotas. Av. Du que de Caxias 250 96030-002, Pelotas RS.

lfachini@terra.com.br.

2 Fundacen tro, Centro

Regi onal da Bahia.

3 Un iversid ade Católica

de Pelotas, Secretaria

Municipal da Saúde

de Pelotas.

4 Un iversid ade Federal

da Bahia.
Abstract This paper presents a review of challenges and perspectives regarding the development of an occupa tional health information system in the Brazilian Unified Health System (SUS). It stress the need to set up a process of continuous data collection, processing, analyzing and communication of information on worker's health and its determinants, emphasizing occupational risks. The paper also address the need to support the training of health workers. It is also recommended to take into considera tion the feasibility of database standardization that allows linkage and, improvement in the information technologies utilized in health services, especially in community health centers, foll owing a coo rd $i$ nated state action. Both, the 3rd National Conference on the Worker's Health, and the federal policy on worker's safety and health have been promoting a debate on the occupational health information system and the need to give priority in the SUS national planning. The proximity of the academic and labor union agendas are pointed as a relevant support to an occupational health information system, that need to be able to strengthen health promotion and preven tion and an effective social pa rticipation in the SUS management organization.

Key words Occupational health, Occupational health services, Information system
Resumo O presente artigo identifica e discute alguns desafios e pers pectivas rel a tivos à implantação de um Sistema de Informações em Saúde do Trabalhador (SIST) no âmbi to do Si s tema Único de Saúde (SUS). Os desafios para a coleta, produção e a análise de dados e a disseminação continuada de informações sobre o estado de saúde dos trabalhadores e seus determinantes são revisados. Há destaque pa ra a necessidade de investimen tos em capa citação de recursos humanos, articulação e harmonização das ba ses de dados de intere s seà saúde do trabalhador, implantação de infra-estrutura de informática nos níveis locais e da coleta das informações na rede de serviços do SUS, e integração e articulação interministerial. A realização da 3 a Conferência Nacional de Saúde do Trabalhador e a recém aprovada Política Nacional de Segu rança e Saúde do Trabalhador representam reforços importantes pa ra o debate sobre a implantação do SIST e sua prioridade na agenda do SUS. A relativa sintonia entre as agendas de técnicos, pesquisadores, trabalhadores e lideranças sindicais na defesa co mum de um sistema de informações também é vista como um apoio à implantação do SIST e seu efetivo controle social.

Palavras-chave Saúde ocupacional, Serviços de saúde ocupacional, Sistemas de informação 


\section{Introdução}

O Brasil ainda é um recordista mundial de acidentes de trabalho, com três mortes a cada duas h oras e três aci den tes não fatais a cada um minuto. Oficialmente, são registrados $390 \mathrm{mil} \mathrm{ac} \mathrm{i-}$ den tes de trabalho por ano no Brasil, com cerca de três mil mortes. Mas o Mnistério da Previdência Social calcula que esse número ch eg u e a 1,5 milhão/ano consideran do todas as ocorrências que deveriam ser cadastradas e não são (ILO, 2005; Brasil, 2003; CUT, 2004).

As doenças ocupacionais representam um ri s co crescente e diversificado para os trabalhadores neste cenário de significativas mudanças tecnológicas, gerenciais, demográficas e de escassez financeira que afetam os processos de trabalho. Problemas músculo-esqueléticos, câncer, perda auditiva, in toxicações químicas, acidentes, doenças cardiocirculatórias e problemas emoci onais são alguns dos mais importantes agravos que os trabalhadores padecem no país e que são passíveis de agravam en to com a alta exploração dos trabalhadores, flexibilização dos con tratos de trabalho e importação de tecnologias sujas, decorrentes da globalização (Laurell et al., 1989; 1991; Fassa et al., 1996; Rego, 1998; Facchini et al., 2003; Santana et al., 2003; 2004; Ribei ro et al., 2004; Faria et al., 2004; Osório et al. , 2005).

Além das condições prévias de vida e saúde, são as cargas de trabalho a que estão submetidos, processos de trabalho insalu bres e perigosos, com equipam en tos e tecnologias defasadas, altos níveis de ruído, ritmo intenso e movimentos repetitivos, en tre tantos fatores, que aumentam os riscos de adoecimen to e invalidez dos trabalhadores (Laurell et al., 1989; 1991; Fassa et al., 1996; Rego 1998; Facchini et al., 2003; Santana et al., 2003; 2004; Ribei ro et al., 2004; Faria et al., 2004; O só rio et al., 2005).

A possibilidade de iden tificar a relação de problemas de saúde com as atividades de trabalho e os riscos derivados dos processos produtivos é crucial para a definição mais adequ ada de priori dades e estratégias de prevenção em saúde do trabalhador. Pa ra isso, um Si s tema de Informações em Saúde do Trabalhador (SIST) é apontado como uma alternativa, que esbarra nas características da con cepção e construção dos sistemas de informação em saúde e das bases de dados de interesse. A implantação do SIST tem alimentado um fértil debate no campo da saúde coletiva no Brasil, com muitos questionamen tos sobre como se produz infor- mações, para que e para quem elas são produzidas, a quem cabe gerir e como se gere a informação (Moraes et al., 1998; 2001; Nobre, 2003), especialmente na sua comunicação para os atores interess ados, a exemplo de outras informações em saúde já veiculadas de modo sistemá tico e com alto grau de qualidade como o Sistema de Informações de Mortalidade.

As demandas indicam a necessidade de um sistema de informação que valorize cada encon tro do trabalhador com o Sistema Único de Saú de (SUS), pri orizando a atenção ao trabalhador em suas ações individuais e coletivas, ta n to preven tivas, qu a n to cura tivas e de re a bilitação. Mas este SIST também precisa articular informações qualificadas sobre as exposições oc u pacionais e aspectos rel eva $n$ tes da vida profissional que determinam e modulam a ocorrência de agravos à saúde do trabalhador (Nobre et al., 1995; Nobre, 2003; Brasil, 2005).

Os estudos acadêmicos, mais específicos e eventuais, muitas vezes conduzidos a partir de in formação de fon te primária, valorizando a ex periência e a informação referida pelo trabalhador, são recursos valiosos no aprofundamento teórico e metodológicona avaliação em saúde do trabalhador. Os seus achados são muito relevan tes para a qualificação das políticas de saúde do trabalhador, e precisam ser financeiramen te apoiados e cri teriosamente utilizados no desenvolvimen to de um Sis tema de Informações em Saúde do Trabalhador (SIST).

Neste artigo refletimos acerca dos desafios relativos à col eta sistem á tica de informação sobre o estado de saúde dos trabalhadores e seus determinantes, com vistas a apoiar a efetivação de um SIST do SUS. Esta informação, proporcionada essencialmente pela rede de serviços de vigilância e de atenção à saúde, precisa oportunizar a detecção precoce da perda de saúde individual, a avaliação dos ris cos ocupacionais e da eficácia das medidas preven tivas, curativas e reabilitadoras. Também deve con tribuir para a melhoria dos cuidados de saúde e para o controle de exposições aos riscos ocupacionais. É, portanto, ferramenta fundamental para o planejamen to e avaliação das ações de saúde, em geral, e de saúde do trabalhador, em particular (Nobre et al., 1995; Rep u llo Jr, 2001; Santana et al., 2005).

Felizmente, o debate sobre o SIST deverá ganhar um maior destaque na agenda do SUS em curto prazo, especialmente por conta da realização da 3a Conferência Nacional de Saúde do Trabalhador. Buscan do estimular o de- 
bate, este artigo também examina algumas perspectivas para a efetivação do SIST no país (Abrasco, 2003; 2004; Ripsa, 2003; CNS, 2003; CNS, 2005; SESRS, 2005).

\section{Desafios na con strução de um Si s tema de Informações em Saúde do Trabalhador no SUS}

O propósito de tornar o SIST prioridade do SUS é altamen te desejá vel e está manifes to em vários documen tos governamentais recentes (Brasil, 2002; 2005; CNS, 2005; Ripsa, 2003). Entretanto, sua materialização depende da superação de desafios significativos, com repercussões teóricas, instrum entais e operacionais, tan to necessárias à produção e gerenciamento da informação, quanto a sua utilização (Mini sté rio da Saúde, 2004; Santana et al., 2005).

O SUS dispõe de uma complexa gama de sistemas de informação em saúde de base individual e abrangência nacional. A natu reza pública, universal e gratuita do SUS permite que s eus sistemas de informação alcancem, s enão a totalidade, boa parte da população de trabalhadores do país. Considerando a baixa cobertura do Segu ro de Aci dentes do Trabalho (SAT) da Previdência Social, menos de um terço da População Econ omicamen te Ativa (Brasil, 2005), o potencial dos Sistemas de Informação em Sa ú de (SIS) para abra n ger as parcelas de trabalhadores inseridas em atividades do mercado in formal de trabalho, servi dores públ i cos e outros é mu i to expres sivo.

En tret an to, na gran de maioria dos municípios e Estados brasilei ros não existe qualquer tipo de SIST vinculado ao SUS. Em boa parte dos casos, os sistemas de informação são precários, se transformaram em uma mera rotina burocrática, e embora os dados sejam coletados não há análise pertinen te para a re s posta a perguntas rel evan tes para os gestores, e não há uma aproximação com os reais problemas de saúde dos trabalhadores. Em compensação, ao lon gode 25 anos de reforma sanitária no país e de 15 anos do SUS, várias experiências municipais e estaduais sobre SIST foram desenvolvidas (Con ceição et al., 2002; Nobre et al., 1995; SES/RS, 2005; SES/SP, 2005). Em alguns municípios e Estados, a tentativa de implantação de um SIST vem sen do frustrada. Probl emas relacionados à ex tensão dos formulários, dificuld ade do regi s tropad ron i zado de ocupação, falta de inform a tização dos serviços e capacitação dos profissionais, dentre outros, foram identificados como as principais razões para esse fracasso (Câmara et al., 2001).

Na medida em que o SUS não valoniza o trabalho como uma das categorias cen trais para o en tendimen to do processo saúde - doença na população, em especial dos determin a ntes, além da grande dimensão das demandas cotidianas de serviços, não é de estranhar que a implantação de qualquer sistema de informação, que implique a coleta sistemática de novos dados acabe não se efetiva ndo. Há um razo á vel consenso sobre a escassez e inconsistência das informações s obre a real situação de saúde dos trabalhadores. Quando analisadas, os dados mostram probl emas de qualidade, especificamen te a não existência de variáveis de interesse para a com preensão do processo saúde/doença do trabalhador, ou o não registro ou sub-registro de variáveis importantes. O utros problemas se referem à pobre qualidade, como a baixa fidedignidade, inconsistências e não pad ronização das variáveis, que levam a dificuldades de harm onização e articulação en tre os diversos sistemas (Cordei ro et al. 1999; Waldvogel, 2002; Ministérioda Saúde, 2004; Santana et al., 2005).

Logo, um desafio estrutural para a implantação do SIST é o registro dos con t a tos dos trabalhadores com o sistema de saúde, incluindo informações sobre o trabalhador e seu trabalho em sistemas, como por exemplo, de mortalidade, de agravos de notificação obrigatória, a tenção básica de saúde e atenção hospitalar, incluindo as urgências e emergências (Nobre, 2003; Santana et al., 2005).

Tod avia, a captação de novos dados esbarra na enorme quantidade de dados coletados e disponíveis, pou co conhecidos, e por isso mesmo pou co utilizados, o que por sua vez con tribui para a piora da sua qualidade (Carvalho, 1997; Moraes et al., 1998; Nobre, 2003; Ministério da Saúde, 2004; Tomasi et al., 2004). A demanda por novos dados aumenta o tempo dedicado à coleta, sem garantir sua análise e divulgação, con tribuindoainda mais para o empobrecimen to da qualidade. A gran de quantidade de dados registrados pelos profissionais de saúde sobre cada indivíduo parece ter pouco significado para suas atividades cotidianas, o utro fator relevante para a manutenção da precariedade das informações produzidas (Nobre, 2003; Tomasi et al., 2004; Moraes et al., 2001).

A fragmentação e a falta de padronização na repre s entação e troca da informação, a irracionalidade e superposição de informações, o 
al to desenvo lvimen to em tecnologias da informação nos níveis cen trais de gestão, e sua precariedade nos níveis de coleta e processamen to da informação, desafiam a lógica e a funcion alidade dos SIS de base nacional no Brasil (Ministério da Saúde, 2004; Brasil, 2005).

Estes problemas também foram observado $s$ em ex periências localizadas de de senvo lvimento de SIST no Brasil. Em um estudo realizado em Minas Gerais, os autores identificaram importantes inadequações na coleta de dados do SIST. As informações em saúde do trabalhador também eram desconhecidas, não sendo utilizadas para subsidiar as ações e políticas sociais (Câmara et al., 2001).

Assim, é preciso parcimônia quando se pensa em estabelecer a obrigatoriedade da coleta sistemática de novas informações em saúde. Especialmente se elas impõem uma dificuldade particular por sua complexidade e dificuldades de registro por uma extensa rede de profissionais de saúde já sobrecarregados com suas tarefas cotidianas. Não há necessidade de se dispor de um único e completo SIST capaz de coletar e processar todas as informações pertinentes à área. Quando se dispõe de informações em larga escala, provenientes de diversos âmbitos do setor saúde, e das exposições vinculadas ao trabalho, o mais razoável parece ser a articulação e padronização das bases de dados, que continuarão cumprindo com suas funções originais (Ministério da Saúde, 2004; Rep ullo Jr., 2001).

O SIST precisa permitir uma adequada transparência da gestão dos serviços, possibilitar um efetivo controle social, atender às nece ssidades de informação dos profissi onais de saúde, prestadores de serviço e ge s tores das três esferas de governo, viabilizando o uso da informação e de sua capacidade de apoiar a tomada de decisão. Não deve se constituir apenas em uma demanda bu rocrática, decorrente apenas de nossa capacidade de demandar dados (Ministério da Saúde, 2004). A inclusão nos sistemas de informações em saúde das variáveis "ramo de ativid ade econômica" e "ocupação" e de suas respectivas tabelas de classificação Classificação Naci onal de Atividades Econ ô micas (CNAE) e Classificação Brasileira de Ocupações (CBO) - em seus níveis máximos de desagregação, requer a articulação e padronização das bases de dados, com ampliação significa tiva de seus propó si tos ori ginais e, talvez, nos custos institucionais. Essa inclusão poderá se tornar factível, desde que apoiada em um pla- no estratégi co de implem entação que con si dere ações de médio e lon go pra zo (Nobre, 2003; Ripsa, 2003; Mnistério da Saúde, 2004).

Uma altern a tiva parci m oniosa seria utilizar o cadastro do Cartão Nacional de Saúde, o conhecido "cartão SUS", como a base de dados inicial do SIST. A portaria/SAS/MS no 174 determinou que os proced im en tos ambulatoriais e hospitalares realizados pelo SUS, que exigem autorização prévia sejam acompanhados, obrigatoriamente, da identificação dos usuários por meio do número do Cartão Nacional de Saúde (Brasil, portaria SAS/MS no 174, 2004). $\mathrm{Na}$ atualidade, talvez mais de 100 milhões de bra sil ei ros já estejam cad a strados, di s pon do de um número de identificação unívoca. Logo, teoricamen te em todo con ta to do usuário com o SUS, desde o atendimento em uma UBS, até a realização de uma cirurgia de alta complexidade, s erá possível buscar no cadastro o número único e inequí voco de cada usuário e obter sua ficha com pl et a.

Na prática, para a plena efetivação deste con ceito, existem grandes problemas, que são relacionados tanto a aspectos operacionais do cad astram en to, quanto à infra-estrutu ra nece ssária para acessar os cadastros el etrônicos. Por exemplo, o programa de cad as tramento do Departamen to de In formação e Informática do SUS (DATA-SUS) não perm $i$ tia a ch ec a gem dos $\mathrm{d}$ ados adequadamente, geran do possibilidade de duplicações e outras inconsistências. Um mesmo indiví duo poderia ser cad a s trado vária s vezes devido ao registro do seu nome com variações ortográficas ou de extensão, no momento do con t a to com o serviço. Es te probl ema pode ter sido agravado em decorrência das exigências da portaria no 174 , que estimulou o novo cadastramen to de indivíduos já registrados, mas que não eram localizados ou não dispunham do número deidentificação. Erros de endereço do indivíduo, especialmente nomes de ruas e bairros, também são um problema que se es tende pelo país, mesmo em grandes centros urbanos. Para a correção da base do cartão SUS, os cadastros deverão ser revisados e checados um a um, en qua n to novos cadastros estão sendo feitos. Em síntese, é necessário um adequado plan ejamento e gestão, como de qual qu ersistema de informações, de modo a se evitar desperdício de tem po e recursos e o con strangimento diantede or ganismos internacionais que rei teram a nece s sid ade da produção e disseminação regulares de informações sobre a saúde do trabalhador. 
En tret an to, a plena utilização da identificação unívoca dos indivíduos para efei to de acesso à base de dados de interesse para a saúde depende da inform a tização dos processos de trabalho em saúde, principalmente das unidades básicas de saúde (UBS). Sua efetivação deverá facilitar a $\mathrm{p}$ ad ronização do registro individual, permitindo o acesso el etrônicoe imedia toa dicionários ou cad a s tros com as informações sobre a va riável de interesse, como por exemplo, Classificação In ternacional de Doenças (CID), CNAE e CBO. A informatização também facilita a definição do conjun to essencial de dados de cada sistema de informação que irá alimentar automaticamen te um outro sistema de interesse. Por exemplo, o registro el etrônico de um agravo de no tificação compulsóra em uma UBS pode alimentar automaticamen teo sistema de vigilância epidemiológica, s em a necessid ade do preen ch imen to de um novo formulário.

No Brasil, a reor ganização da atenção básica através do Programa de Saúde da Família prevê o trabalho de equi pes de saúde em territó rios definidos, com adscrição da dien tela, de m odo articulado com os agen tes comunitários de saúde que, com base em mapa de risco do território, irão organizar suas ativid ades. Este m odelo prevê a utilização da ep i dem i o logia para subsidiar as prá ticas de saúde, in corporando a pers pectiva da vigilância em saúde. Sen do este territó rio o local de en con tro de trabalhadores da saúde com a população trabalhadora, coloca-se o desafio de pensar um sistema de informação em saúde do trabalhador articulado ao sistema de informação da atenção básica (SIAB) e ao sistema de informação ambulatorial (SIA), que monitore acidentes e agravos neste nível da atenção, com possibilidade de captar dados de trabalhadores vinculados principalmente ao setor informal, aumentando assim a abrangência do SIST (Nobre, 2003; Ministério da Saúde, 2004)

A informatização também permite articular diferentes níveis de detalhamen to ou agregação de um mesmo ti po de informação, como a CNAE e a CBO, subordinando as diferentes bases de dados a um padrão hier á rqui co único. Desta maneira, será mais fácil en f rentar o desafio do conhecimento das populações, sejam as gerais, de trabalhadores, como as específicas para categorias de trabalhadores, que são comumen te utilizadas como denominadores em estudos sobre a saúde do trabalhador. No mo$\mathrm{m}$ en to, essas informações não são disponíveis, especialmente para o expres sivo segmento dos trabalhadores informais. Para os empregados de em presas de médio e gra n deporte, o desafio s erá articular informações provenientes dos cadastros das em presas com número de funcionários, da Relação Anual das In formações Sociais (RAIS) e de relações forn ecidas por sindicatos de trabalhadores. Por outro lado, a articulação das informações provenientes das bases de dados censitárias com as de pesquisas amostrais, a exemplo das desenvolvidas pelo Instituto Brasilei ro de Geografia e Estatística (IBGE) e o Departamen to In tersindical de Estatísticas e Estu dos Socioecon ô mi cos (Dieese), pode ser especialmen te rel eva $n$ te para iden tificar trabalhadores de micro e pequ enas em presas, autôn omos e informais (Rep u llo Jr., 2001).

A informatização dos serviços de saúde, principalmente das unidades básicas de saúde, é um desafio primordial para um avanço da qualidade do atual estágio de des envo lvim en to dos SIS, não apenas em municípios men ores e mais pobres. Nos municípios maiores, geralmente o nível central ou administrativo do SUS dispõe de um acesso relativamente bom aos recursos de tecnologia da informação, mas o registro das ações dos serviços de saúde ainda é, majoritariamente, manual. Assim, é preciso informatizar o processo de coleta de informações, facilitando o registro único da informação rel evan te para diferen tes etapas do processo de trabalho em saúde, evitando repetições de ro tinas de trabalho em especial das et apas de digitação e processamento manual. $\mathrm{Ou}$ seja, é possível pensar no uso de regi stros on-li$n e$, no qual o país tem larga experiência em processamento de complexos sistemas de informação. Outro probl ema passível de melhoria sign i fi c a tiva com a inform a tização é o regi stro dos agravos de notificação compulsória. Se a notificação manual de doenças clássicas e de critérios diagnósticos consensuais, como por exemplo, caxumba e varicela em crianças, é problemática, imagine-se o sub-registro de problemas de saúde de difícil diagnósti co como, por exemplo, intoxicações por agrotóxicos e Lesões por Esforços Repetitivos (LER/DORT) (Ministéro da Saúde, 2004). Some-se ainda, as implicações legais, que vão des de multas pesadas a responsabilidade jurídica e prejuízos na própria imagem da empresa com a revelação de probl emas de saúde que podem ser graves e são amplamen te reconheci dos como evitáveis.

A alimentação direta das bases também possibilita o processamen to automático dos dados e a produção imediata de análises úteis à 
tomada de decisão e no apoio à prática profissional. Nas ações típicas de saúde coletiva como a Vi gilância Sa nitá ria, Vigilância Ep i demi ológica, Vigilância Ambiental e Vigilância da Saú de do Trabalhador, en tre outras, quase inteiramen te baseadas em registros manuais, a in formatização deverá proporcionar ganhos de eficiência equalidade na realização das atividades e maior fidedignidade nas informações regis tradas (Ministério da Saúde, 2004).

Entret a $n$ to, os ben efícios das tec nologias da in formação para o SIST também podem contribuir para a devassa e o controle minucioso da vida do trabalhador, capaz de romper com a privacidade e os direitos individuais, permitindo, portanto, a quebra de princípios da ética profissional. Portanto, a ênfase nos aspectos de confidencialidade e segurança da informação individual iden tific ada repre senta mais que um desafio, mas um compromisso ético fundamental, que certamen te será cumprido pelo SUS (Ministério da Saúde, 2004).

Por outro lado, pa ra a viabilização do SIST não basta som en te o acesso à tecnologia da informação. A utilização de SIS deve considerar, além da dimensão tecnológica, seus métodos, recursos humanos e recursos organizacionais com fins de maximizar a utilização dos res pectivos sistemas (Câmara et al., 2001).

$\mathrm{O}$ su cesso e a qualidade de registros de interesse do SIST, como, por exem plo, a CNAE e a CBO, demandam um abra nen te processo de capaditação e apoio à prática profissional, poi s os indicadores captados pelos sistemas de informação são meros reflexos de uma situação real. Por con seguinte, medidas indiretas e parciais de uma situação com pl exa são passíveis de reducionismos, erros e distorções. Se calculados seqüencialmente, no tempo, podem indicar a direção e a velocidade das mudanças e s ervem para comparar diferen tes áreas ou grupo de pessoas em um mesmo mom en to (Mello Jor ge et al. , 2001).

A divulgação, monitoramen to e avaliação de informações em saúde do trabalhador e de intervenções em benefício da saúde do trabalhador representam outro desafio essencial à implantação do SIST, com importantes repercussões na capacitação de recursos humanos e na utilização da informação para a tomada de decisão.

\section{Perspectivas de efetivação do SIST no SUS}

A magnitude e a complexidade dos desafios do SIST deixam antever perspectivas bastante limitadas para sua implantação em curto pra zo. Entretanto, um olhar mais aten to é capaz de captar indícios de que o SIST poderia ser factível em um prazo não muito lon go e com um custo aceitável (RIPSA, 1998; 2003; Nobre, 2003).

A história da saúde do trabalhador no SUS e na área de saúde coletiva tem sido construída em forte aliança com os trabalhadores e os movimen tos sociais, que se ex pressa em experiências e pesquisas apre en tadas nos con gressos de saúde coletiva, conferências, even tos, con ferências de saúde e outros fóruns de participação social. Há uma sintonia bastante ra zoável en tre as agendas atuais de gestores, pesquisadores e profissionais de saúde, e as demandas de trabalhadores e lideranças sindicais em relação ao SIST. Logo, há uma possibilidade efetiva de articular o apoio dos trabalhadores e dos movimentos sociais do país, no transcurso da $3 \underline{a}$ CNST, com vistas a agilizar a efetivação do SIST em um futuropróximo.

O relatório final da Oficina de Trabalho sobre "Políticas de Saúde do Trabalhador no SUS”, realizada durante o VII Congresso de Saúde Coletiva, em 2003, con clui pela necessidade de se construir um sistema nacional de in formação em saúde do trabalhador. O relatório apre s enta várias proposições rel eva n tes para o SIST, com destaque para a necessidade de o timizar as informações dos bancos já exis tentes (SIM, SIAB, SINAN, SINITOX, SIH, etc.), além de retomar as relações e articulações interinstitucionais (Ministérios do Trabalho e Emprego; Fund acentro, Previdência Social); a necessidade de registrar condições de saúde e não só agravos ou riscos com informações ágeis e descentralizadas; a necessidade de pautar a questão da informação em saúde do trabalhador como um tema específicoa ser discuti do e aprofundado e a necessidade de se construir um sistema nacional de informação em saúde do trabalhador (SIST) para a orientação e otimização das ações de vigilância em Saúde do Trabalhador (Abrasco, 2003).

Essas demandas expressam a maturidade do debate sobre o SIST, em grande parte fruto de várias ex periências acumuladas em municípios e Estados brasileiros, a partir da implantação de políticas de saúde do trabalhador, cen- 
tros de referência em saúde do trabalhador e, inclu sive, de sistemas de informação em saúde do trabalh ador (SES/SP, 2005; Conceição et al., 2002; SES/RS, 2005). Durante a década de 1990, d iversos Estados e municípios incluíram agravos à saúde do trabalhador em portarias que atualizaram sua relação de agravos de notificação compulsória (Brasil, 2002). Mas, dificuld ades e de s con ti nu id ades ob s ervadas na implementação do SIST, como por exemplo, no Rio Gra ndedoSul, que ch egou a implantar obs ervatórios de aciden tes e violências em hospitais e serviços de emergência, mostram a vulnerabilidade de iniciativas locais e sua forte dependência de compromissos governamentais (SES/RS, 2005). O utros Estados também tiveram experiências similares, com dificuldades de inserir a vi gilância e a notificação dos agravos relacion ados ao trabalho nas práticas, rotinas e normas da vigilância epidemiológica, com descontinuidades e mudanças de diretrizes políticas.

Espera-se que a recen te portaria federal no 777/GM, publicada pelo Ministério da Saúde em 2004, que dispõe sobre os procedimentos técnicos para a notificação compulsória de agravos à saúde do trabalhador em serviços sentin ela específicos, possa impulsionar a consolidação dessas experiências nos Estados e municípios (Brasil, 2004). Mais do que grandes definições, o investimen to em capacitação de recu rsos humanos na ponta e em setores estratégicos do sistema estadual e municipal de saúde é neces sá rio para ga ra $n$ tir esses avanços.

Dentre os fatos positivos e alentadores pode-se afirmar que, nos últimos anos, houve um incremento de estu dos e da produção científica, que têm priorizado a análise da qualidade das informações em relação aos indicadores de saúde do trabalhador, seja mediante a adoção de metodologias de comparação de diferentes bases ou fon tes de dados (da Previdência, de mortalidade, hospitalar, registros policiais, etc.) (Carneiro, 2000; Pepe, 2002; Waldvogel, 2002; Souza, 2003; Con ceição et al., 2003), seja com estratégias de reconstrução de causa básica de ó bi to (ou de morbi d ade) (Cordei ro et al., 1999; Hennington et al., 2004) ou de estimativas de sub-registro em abordagens populacionais (Barata, 2000; Santana et al., 2003; Nobre, 2003).

De outro lado, existem algumas ações federais relacionadas aos sistemas de informação em saúde que facilitam as demandas do SIST. A i dentificação unívoca de cidadãos, particular- mente dos usuários do SUS, a padronização e articulação de bases de dados e a informatização dos serviços de saúde, oportunizandoo registro eletrônico de dados, representam um impulso bastante favor á vel para a implantação do SIST (Brasil, 1998; 2001; Ministério da Saúde, 2004).

A implantação da Rede Nacional de Atenção à Sa ú de do Trabalhador (RENAST) (Brasil, 2002), a publicação da Política Naci onal de Segurança e Saúde do Trabalhador (PNSST) (Brasil, 2005) e a realização da 3a Conferência Nacional de Saúde do Trabalhador (CNST) (CNS, 2005) também fortalecem as perspectivas do SIST. O Plano Estadual de Sa ú dedo Trabalhador, estabelecido conforme a RENAST (An exo I da portaria 1.679/2002), com rotinas de sistematização, processamen to e análise dos dados sobre saúde do trabalhador, gerados nos municípios e no seu próprio campo de atuação e de alimentação regular das bases de dados estaduais e municipais, poderá ser um piloto do SIST. A el a boração do perfil epidemiológico da saúde dos trabalhadores no Estado, a partir de fon tes de informação e, se necessário, por intermédio de estu dos específicos, poderá subsidiar a programação e avaliação das ações de atenção à saúde do trabalhador, tornando-se essencial para o de senvo lvimen to do SIST. Da mesma forma, a instituição e manutenção de cadastro atualizado das empresas, dassificadas nas atividades econômicas desenvolvidas no Estado, com indicação dos fatores de risco a que está exposto o contingen te populacional, direta ou indireta mente.

A promoção de ações em saúde do trabalhador articuladas com outros setores e instituições, tais como a Previdência Social, Ministério do Trabalho e Emprego, Fundacentro e Sindicatos, juntamen te com a implementação da notificação dos agravos à saúde, na rede de atenção do SUS, e de ri s cos rel aci on ados com o trabalho serão altamen te rel eva n tes para a efetivação do SIST. A alimentação regular das bases de dados de interesse da saúde do trabalhador, a ro tina de sistem a tização e análise dos dados gerados na assistência à saúde do trabalhador, as intervenções ori en $\mathrm{t}$ adas da vigilância e a organização das ações em saúde do trabalhador são ações estratégicas para a efetivação do SIST. Os programas de educação permanente, acompanhamen to e avaliação, aliados a ações sistem á ticas de vigilância em ambientes e processos de trabalho também con correm positivamente para o SIST. 
A Portaria In terministerial no 800/2005, que propõe a Política Nacional de Segurança e Saúde do Trabalhador, deu um grande passo em direção à superação da fragmentação, desarticulação e superposição das ações implementadas pelos setores Trabalho, Previdência Social, Saúde e Meio Ambiente. Sua efetivação é uma ação de governo fundamental para a á rea de saúde do trabalhador e poderá facilitar bastante a implantação do SIST. Con cebida como um esforço interministerial, que está diretamen te relacionada com as políticas dos $\mathrm{Mi}$ nistérios do Trabalho e Emprego, da Previdência Social, do Meio Ambien te e da Saúde, a PNSST apresenta interfaces com as políticas econômicas, de Indústria e Comércio, Agricultura, Ciência e Tecnologia, E ducação e Justiça, em uma pers pectiva inters etorial e de tra n s versalid ade (Brasil, 2005).

A PNSST trata a informação como ponto vital da política de seg u rança e saúde do traba$1 \mathrm{~h}$ ador que preten de implantar. Ao propor a estruturação de uma rede integrada de informações em saúde do trabalhador que permita o compartilham en tode seu uso en tre os ministérios envolvidos, a PNSST destaca as seguintes es tratégias (Brasil, 2005):

- Pad ronizar os con cei tos e critérios qu a n toà con cepção e caracterização de ris cos e agravos à segurança e saúde dos trabalhadores relacio$\mathrm{n}$ ados aos processos de trabalho.

- Compatibilizar os Sistemas e Bases de Dados, a serem partilhados pelos Ministérios do Trabalho e Emprego, Previdência Social, Mei o Ambi en tee Saúde.

- Compatibilizar os instrumen tos de coleta de dados e fluxos de informações.

- Insti tuir a concepção do nexo epidemiológico presumido para aciden tes e doenças relacion adas ao trabalho.

- Atri buir ao SUS a competência de estabel ecer o nexo etiológi co dos acidentes e doenças relacionados ao trabalho e analisar possíveis questionamentos rel acion ados com o nexo ep idemiológicopresumido.

- In cluir nos Sistemas e Ban cos de Dados as informações contidas nos relatórios de intervenções e análises dos ambien tes de trabalho, elaborados pelos órgãos de governo envo lvidos nesta Política.

- Desenvo lver um amplo programa de capacitação dos profissionais, para o desenvolvimen to das ações em segurança e saúde do trabalhador, abrangen do a promoção e vigilância da saúde, prevenção da doença, assistência e reabilitação, nos diversos espaços sociais on de essas ações ocorrem.

A 3a Conferência Nacional de Saúde do Trabalhador, cuja etapa nacional ocorrerá de 9 a 12 de novembro de 2005, também será uma oportunidade de fortalecer o debate e as demandas em torno da efetivação do SIST. O documento que serve de referência histórica às discussões e deliberações das etapas municipal, e s t adual e nac i onal reafirma a responsabilidade do Si s tema Único de Sa ú de com a vigilância da saúde dos trabalhadores, assim como da integração das políticas internas da saúde que afetam o trabalho. O tex to assume, como tarefa dos sistemas de informação do SUS, a compilação das informações relacionadas ao atendimen to de trabalhadores acidentados e portadores de doenças do trabalho. Como os sistemas de informação do SUS incluem os trabalhadores informais, o SIST conforma uma grande con tri buição para a redução significativa da subnotificação de acidentes e agravos de saúde no trabalho. Igualmente, há uma ênfase na formação e capacitação de recursos humanos e a implem entação de um sistema de informatização e de comunicação ágeis, que integre os diversos setores envo lvi dos nas políticas públicas de saúde do trabalhador (Brasil, 2005).

Emsuma a 3a CNST é um importante espaço de participação social e poderá acel erar a efetivação do SIST. Con forme o regimen to e os documen tos de apoio, o SIST será debatido nas etapas municipais, e st aduais e nac i onal da Conferência, em função de sua centralidade para articular repostas às questões antepostas pelos três ei xos do temário: 1) Como garantir a integralidade e a transversalidade da ação do Estado em saúde dos (as) trabalhadores (as)? 2) Como incorporar a saúde dos (as) trabalhadores (as) nas poli ticas de desenvolvimen to sustentável no País? 3) Como efetivar e ampliar o co n trole so cial em saúde dos (as) trabalhadores (as)? (Brasil, 2005).

Assim, do pon to de vista do SUS, a bre-se a possibilidade de divulgar e fortalecer a agenda de pri oridades do SIST, através do debate e do apoio dos participantes das etapas municipais, estaduais e nacional da 3a CNST (CNS, 2005). O com promisso de não se implantar novos sistemas de informação, que simplesmente aumentam a carga de trabalho dos profissionais de saúde, diminuindo o tempo dedicado à tarefa prim ordial de atenção à saúde fortalecerá o a poio ao SIST. Pa ra isso, deve-se buscar apoio a uma política de saúde do trabalhador que prom ova e viabilize, de modo articuladb e referen- 
ciado, a integração das bases de dados de interesse da saúde do trabalhadbr, a capacitação de recursos humanos envolvi dos com a saúde do trabalhador, a informatização e modemização da tecnologia de captação e processamen to da informação em saúde do trabalhador, a análise sistemática de informação em saúde do trabalh ador e a devo lução da informação aos profissionais de saúde responsáveis por sua produção.

As deliberações da 3a CNST precisam fortalecer a implantação da PNSST e, assim, do SIST. Nes te processo, é necessário va l orizar instituições e organismos habilitados a produzir estudos em profundidade sobre as bases de dados disponíveis, identificando seus problemas e suas potencialidades para revelar perfis e tendências em saúde do trabalhador. Um projeto nacional liderado pelo SUS, com a necessária articulação interministerial e interinstitucional, deve prom over a efetivação do SIST.

Partin do da con cepção de um proj eto integrado, poderiam ser articulados e integrados quatro projetos específicos, precedidos da necessária pactuação interge stores e com o propósitode viabilizar a implantação da PNSST e a efetivação das principais portarias ministeriais que dispõem sobre informações em saúde (do trabalhador) (portarias no 3.947/1998, 1.969/2001, 777/2004). Primeiro, de s tacamos a implementação de um projeto de articulação das bases de dados de interesse da saúde do trabalh ador, cujo objetivo seria conduzir a padronização das bases de dados do SUS, MPAS, MTE, IBGE, base estrutu ral para a implantação do SIST. Segundo, um amplo projeto de capacitação de recursos humanos em saúde do trabalhador, com ênfase para o SIST, dirigido a gestores e profissionais de saúde do SUS nas três esferas de govern o, além de representantes do MPAS, MTE, IBGE e insti tuições de ensino e pesquisa, de forma a efetivar a padronização e qualificação das bases de dados de interesse. Um apoio estratégi co para a capacitação e, em conseqüência, a viabilização do SIST, será um proj eto de inform a tização e modernização tecnológica destinado prioritariamen te às unidades básicas de saúde, Cen tros de Referência em Saúde do Trabalhador e setores de vigilância à saúde e informação em saúde de estados e municípios. Finalmente, para completar este processo de st aca-se um proj eto de monitoramento e disseminação da informação em saúde do trabalhador, com o prop ó si to de realizar a avaliação do SIST, análises sistemáticas das bases de dados, apoio à prática profissional e ao uso da informação, d ivul gação e publicação con tinu ada de re sultados.

\section{Conclusão}

Impulsi onar a ação govern a m ental em favor do SIST representa um ponto de destaque da agenda da área de saúde do trabalhador, especialmen te com vistas à $3 \underline{a}$ Conferência Nacional de Saú de do Trabalhador e da implementação da Política Naci onal de Segurança e Saú de do Trabalhador.

Compartilh a ndo do pensamento de que Informação em Saúde é um espaço estratégi co para a consolidação da democracia política e da produção e apropriação do saber, e que o acesso ao conhecimento se constitui um dos alicerces do projeto de conquistas sociais e de construção da cidadania (Moraes et al., 1998; 2001), defen demos que a oferta de informação adequ ada sobre questões rel a tivas ao trabalho e ocupação - categorias indicadoras de uma dimensão de fundamental importância na vida das pessoas e das coletividades - de forma que estas possam ter visibilidade nas análises da situação de saúde, significa aumentar o espectro de possibilidades de intervenção e ampliar as possibilidades de participação social e democra tização de nossas práticas (Nobre, 2003). A produção atual de conhecimen to sobre as desigualdades e iniqüidades sociais não pode prescindir da análise dessa dimensão da vida social.

\section{Colaboradores}

LA Facchini e LC Costa Nobre participaram da estrutu ração do tex to, redação final e demais etapas da el a boração do artigo. NMX Faria, AG Fassa, E Thumé, E Tomasi e V Santana parti ci p a ram da re visão bibliográfica e do tex to, acrescentando contribuições à versão final do artigo. 
Abrasco (Associação Brasileira de Saúde Col etiva) 2003. Oficina de trabalho Políticas de Saúde do Trabalhador no SUS. VII Congresso Brasileiro de Saúde Col etiva. Brasília.

Abrasco (Associação Brasileira de saúde Coletiva) 2004. VI Congresso Brasileiro de Epidemiologia. Recife.

Barata RB 2000. Aciden tes de trabalho referi dos por trabalhadores moradores em área urbana no interior de São Paulo em 1994. IESUS 9(3):199-210.

Brasil, Mnistério da Previdência e Assistência Social 2003. Anuário Estatístico de Acidentes do Trabalho. MPAS, Brasília.

Brasil, Mnistério da Saúde 1998. Portaria Federal no 3.947/ MS/GM, de 25 de novembro de 1998. Aprova os a tributos comuns a serem adotados, obriga toriam ente, por todos os sistemas e bases de dados do Ministério da Saúde, a partir de 1o de janei ro de 1999. MS, Brasília.

Brasil, Mnistério da Saúde 2001. Portaria Federal no 1.969/MS/GM, de 25 de outu b rode 2001. Dispõe sobre o preenchimento de Autorização de In ternação Hospitalar - AIH, em casos de quad ro compatível com causas ex ternas e com doenças e aci den tes relacionados ao trabalho. MS, Brasília.

Brasil, Mnistério da Saúde 2002. Portaria Federal no 1.679, de 19 de se tem bro de 2002. Aprova a constitu ição da Rede Nacional de Atenção à Sa ú de do Trabalhador (RENAST). Brasília.

Brasil, Mnistério da Saúde. 2004. Portaria Federal no $777 / G M$, de 28 de abril de 2004. Dispõe sobre os procedimen tos técnicos para a notificação compulsória de agravos à saúde do trabalhabr em rede de serviços sen ti n ela específica, no Si s tema Único de Sa ú deSUS. Brasília.

Brasil, Mnistério do Trabalho e Emprego, Mnistério da Previdência e Assistência Social \& Ministério daSaúde 2005. Portaria Interministerial no 800, de 3 de maio de 2005. Aprova a Política Naci onal de Segurança e Saúde do Trabalhador. MTE, Brasília.

Câ m a raGR\& Pinhei ro TMM 2001. Percepção social dos problemas relacionados com o uso dos sistemas de informação em saúde do trabalhador no Sistema Único de Saúde (SUS). Revista IP - Informática Pública 3(1):111-126.

Carneiro SAM 2000. Trabalho e violência: relação de proximidade da violência a trabalhadores durante a jornada de trabalho, na Zona No rte de São Paulo, em 1998. Dissertação. Universidade de São Paulo, São Paulo.

Ca rvalho DM 1997. Gra n des sistemas nacionais de informação em saúde: revisão e discussão da situação atual. IESUS 6(4):7-46.

CNS (Conselho Nacional de Saúde) 2003. 12a Conferência Nacional de Saúde. Relatório final. Brasília.

CNS (Conselho Nacional de Saú de) 2005. 3- Conferência Na cional de Saúde do Trabalhador. Texto base. Brasília.

Con ceição PSA, Na scim en toIBO, Ol ivei ra PS \& Cerqu eira MRM 2003. Acidentes de trabalho atendidos em serviço de emergência. Cadernos de Saúde Pública 19(1):111-17.

Conceição PSA \& Nobre LCC. 2002. Sistemas de informação em saúde do trabalhador. In Secret a ria da Saúde do Estado da Bahia, Superintendência de Vigilância da Saúde \& Centro de Estudos da Saúde do Trabalha- dor. Manual de Normas e Procedimentos Técnicos para a Vigilância da Saúde do Trabalhador. SESAB, Sa lvador.

Cordei ro R et al. 1999. Valid adedas informações ocupação e causa básica em dedarações de óbi to de Bo tucatu. São Paulo. Cadernos de Saúde Pública 15(4):71928.

CUT 2004. Informativo do Coletivo de Saúde. Brasília, DF.

Facchini LA, Fassa AG, Dall'Agnol MM \& Maia MFS 2003. In dividuals at risk: the case of child labor, pp. 52-71. In J Heymann (org.). Global inequalities at work. Oxford University Press, New York.

Faria NMX, Facchini LA \& Fassa AG 2004. Trabalho rural e intoxicações por agrotóxicos. Cadernos de Saúde Pública 20(5):1298-308.

Fassa AG, Facchini LA \& Dall' Agnol MM 1996. Trabalho e morbidade comum em indústria de celulose e papel: um perfil segundo setor. Cadernos de Saúde Pública 12(3):297-307.

Hennington EA, Cordei ro RC \& Morei ra Filho DC 2004. Trabalho, violência e morte em Campinas, São Paulo, Brasil. Cadernos de Saúde Pública 20(2):610-617.

ILO (In tern a tional Labor Organization) 2005. World day of safety an health at work. 28 Annual Event.

Laurell AC \& Noriega M 1989. Processo de produção e saúde: tra balho e desgaste operário. Hucitec, São Paulo.

Laurell AC, Nori ega M, Arellano OL \& Alcantara SM 1991. El trabajo como determinante de la en fermedad. Cuadernos Médico Sociales (56):17-33.

Mello Jor ge MHP, GotliebSLD \& Lauren ti R 2001. A saúde no Brasil: análise do período 1996 a 1999. OPS, Brasília.

Ministério da Saúde 2004. Política Nacional de Informação e Informática em Saúde. MS, Brasília.

Moraes HIS \& Sa n tos SRFR 1998. In formação em saúde : os desafios continuam. Ciência \& Saúde Coletiva 3(1):37-51.

Moraes HIS \& Santos SRFR 2001. Informações para a gestão do SUS: necessidades e perspectivas. IESUS $10(1): 49-55$.

Nobre LCC 2003. Uso da ocupação e ramo de atividade econômica nos sistemas de informações em saúde: potencialidades e factibilidade. Ciência \& Saúde Coletiva 8(supl. 2): 158 .

Nobre LCC \& Freitas CU. 1995. Si stema de info rmação em saúde do trabalhador no SUS: proposta de sistema de informação de riscos e danos no trabalho a partir do nível local. Ministério da Saúde-OPS, Brasília.

Osório C, Mach ado JMH \&Mi n ayo Gomez C 2005. Proposição de um método de análise coletiva dos acidentes de trabalho no hospital. Cadernos de Saúde Públ ica 21(2):517.

Pepe CCCA 2002. Estratégias pa ra superar a desinformação: um estudo sob re os aciden tesde tra balho fatais no Rio de Janeiro. Dissertação de mestrado. Escola Nacional de saúde Pública, Fundação Oswaldo Cruz, Rio de Janeiro.

Rego M 1998. Non-Hodgkin's lymphoma risk derived from ex po su re to or ganic solven $\mathrm{t} s$ : a revi ew of ep i demiologic studies. Cad ernos de Saúde Pública 14 ( supl. 3):41-66.

Repullo Jr R. 2001. A municipalização das ações de saúde do trabalhador no Sistema Único de Saúde do Brasil. Fundación Mexicana para la Salud, México. 
Ribei ro FSN \& Wünsch Filho V 2004. Avaliação retro spectiva da exposição ocupacional a cancerígenos: a bord a gem epidemiológica e aplicação em vigilância em saúde. Cadernos de Saúde Pública 20(4):881-890.

RIPSA (Rede In tera gencial de In formações para a Saúde) 1998. Relatório do Comitê Seguro, Trabalho e Saúde. Ministério da Saúde, Brasília.

RIPSA (Rede In tera gencial de In formações para a Saúde) 2003. Uso da CBO e CNAE nos sistemas de informação em saúde: potencialidades e factibilidade. Minist ério da Saúde OPS, Brasília.

Santana VS, Maia AP, Carvalho C \& Luz G 2003. Incidência de ac i d en tes de trabalho não fatais: di ferenças de gênero e tipo de contra to de trabalho. Cadernos de Saúde Pública 19(2):481-492.

Santana VS \& Nobre LCC 2005. Sistemas de informações em saúde do trabalhador. 3a Con ferência Nacional de Saúde do Trabalhador.

Santana VS \& Oliveira RP 2004. Saúde e trabalho na construção civil em uma área urbana do Brasil. Cadernos de Saúde Pública 20(3):797-811.

SES/RS (Secret a riaEstadual da Sa ú de - RS) 2005. Si stema de informação em saúde do trabalhador. SES, Porto Alegre.

SES/SP (Sec ret a ria Estadual da Sa ú de - SP). 2005. Centro de referência em saúde do trabalhador. SES, São Paulo.

Souza NSS 2003. Aciden tes de trabalho com óbi to regi strados nas dedarações de óbito no Estado da Bahia no ano de 1998. Cadernos de Saúde do Trabalhador 1:40-43.

Tomasi E, Facchini LA \& Maia MFS 2004. Health information technology in primary health care in developing countries: a litera tu re revi ew. Bulletin of World Health Organization 82(11):867-874.

Waldrogel BC 2002. Aciden tes do tra balho: os casos fatais. A questão da iden tificação e da men su ra çã o. Segraced, Belo Horizonte.

Artigo apre s en tado em 18/07/2005

Aprovado em 10/08/2005

Versão final apre sen tada em 10/08/2005 